International Journal of Medical Sciences

ISSN 1449-1907 www.medsci.org 2008 5(4):203-208

Short Research Communication

(C) Ivyspring International Publisher. All rights reserved

\title{
Contrast-Enhanced Ultrasonograpic Findings in Pancreatic Tumors
}

\section{Chiara RECALDINI, Gianpaolo CARRAFIELLO, Elena BERTOLOTTI, Maria Gloria ANGERETTI, Carlo FUGAZZOLA}

\author{
Department of Radiology, University of Insubria - Viale Borri 5721100 Varese, Italy \\ Correspondence to: Chiara Recaldini MD, Department of Radiology, University of Insubria, Viale Borri 57, 21100 VARESE (Italy). Tel \\ 0039 / 0332 / 278056; Fax 0039 / 0332 / 263104; E-mail: chiarec@libero.it
}

Received: 2008.03.29; Accepted: 2008.07.06; Published: 2008.07.09

OBJECTIVE: The purpose of this article is to present the potentials and limits of contrast-enhanced ultrasonography (CEUS) in the characterization of pancreatic tumors, usually hypoechoic or cystic at B-mode ultrasound. CONCLUSION: As regards hypoechoic lesions at B-mode ultrasound, CEUS often can distinguish among adenocarcinoma, islet cell tumor and serous microcystic adenoma. As regards cystic lesions, CEUS in most cases doesn't add significative diagnostic information; therefore CT, MR or endoscopic US are almost always necessary for their proper characterization.

Key words: contrast-enhanced ultrasonography, pancreatic tumors

\section{Introduction}

Imaging of pancreatic tumors takes advantage of various techniques, such as B-mode ultrasound (US), computed tomography $(\mathrm{CT})$ and magnetic resonance (MR).

B-mode US allows the detection of focal lesions, even small ones about $1 \mathrm{~cm}$ in diameter, that usually are hypoechoic or cystic. However, within hypoechoic lesions B-mode US is not able to differentiate adenocarcinoma (the most frequent tumor), islet cell tumor or other more rare diseases (microcystic adenoma; focal pancreatitis). Similarly, within cystic lesions B-mode US is not often able to differentiate pseudocyst, serous cystadenoma, mucinous cystic neoplasm $(\mathrm{MCN})$, intraductal papillary mucinous tumor (IPMT).

The aim of our pictorial essay is to illustrate the potentials and limits of contrast-enhanced ultrasonography (CEUS) in the characterization of focal pancreatic lesions.

\section{CEUS technique}

CEUS, by using a blood pool contrast agent, can provide dynamic information concerning macro- and micro-circulation of focal lesions and of normal parenchyma [1]. In our experience, all CEUS examinations were performed on a Technos MPX ultrasound system (Esaote Biomedica, Genova, Italy), with harmonic microbubble specific imaging (CnTI: Contrast Tuned Imaging) with low acoustic ultrasound pressure
(MI: 0,093-0,157). A 2,4 ml bolus of second generation contrast agent (Sonovue, Bracco, Milano, Italy) was injected intravenously, followed by a $10-\mathrm{ml}$ bolus of saline solution.

All images were stored on the ultrasound machine as 3 clips lasting 60 seconds each; the dynamic observation of the contrastographic phases (early arterial, arterial, pancreatic, portal and late phases) was possible by maintaining the same scanning frame rate of the B-mode examination.

\section{CEUS appearance}

\section{1) HYPOECHOIC TUMORS AT B-MODE US}

Focal lesions that are hypoechoic at B-mode US can be classified at CEUS as hypoechoic, isoechoic, hyperechoic, according to their enhancement compared to that of the surrounding parenchyma.

a) Ductal adenocarcinoma is usually hypoechoic (absent or poor enhancement compared to normal pancreatic parenchyma), because of its scanty vascularization [2] (Fig. 1). In particular, tumors in which the size of the hypoechoic area is unchanged on CEUS have clear margins with no infiltration or inflammation; tumors in which the size of the hypoechoic area is reduced on CEUS have blurred margins with infiltration of cancerous cells and inflammation. CEUS may be a tool for evaluating pathologic changes of pancreatic cancer and may provide useful information in the pre-treatment phase [2]. 
Ductal adenocarcinoma is rarely isoechoic (enhancement similar to normal pancreatic parenchyma); this pattern is due to a moderate vascularization (Fig. 2) occurring in some histotypes (anaplastic carcinoma and acinar cell carcinoma) [3]. The isoechoic pattern is often observed in focal pancreatitis too [4]: therefore, CEUS is not always able to accurately differentiate adenocarcinoma from focal pancreatitis, as well as CT and MR [5].

Ozawa et al [6] studied 25 pancreatic mass lesions with CEUS and found out that 5/20 adenocarcinoma didn't show any enhancement in the arterial phase and among these only 5 showed a peripheral enhancement in the late phase; on the contrary, all inflammatory lesions showed a mild enhancement. The amount of necrosis, inflammation and fibrosis is the anatomo-pathological substrate responsible for different pattern of vascularization and therefore problems of differential diagnosis [7]. Whereas edema and inflammation requires vascular supply, when fibrosis increases during chronic pancreatitis vascular supply diminishes and subsequently contrast enhancement is less vivid. For example, in autoimmune pancreatitis the enhancement is significant in the early phase, though inhomogeneous, due to lymphocytes infiltrate and perivessel fibrosis.

D'Onofrio et al [4] studied 173 patients affected by pancreatic masses: they could reach a correct diagnosis of focal pancreatitis in 35 cases (19 alcohol-related, 15 autoimmune, 1 genetic) with a sensibility of $88.8 \%$, specificity of $97.8 \%$, positive predictive value of $91.2 \%$, negative predictive value of $97.1 \%$ and accuracy of $96 \%$.

b) Islet cell tumors- functioning or nonfunctioning - are usually hyperechoic in the arterial phase (enhancement superior to normal pancreatic parenchyma) because of their rich vascularization [8]; after the enhancement a rapid washout of the lesion is appreciable, resulting in a slightly hypoechoic appearance during the venous phase [8]. Sometimes, enhancement is more important in the portal phase (Fig. $3)$, similarly to what has been reported for dynamic studies performed with CT [9]. In addition, in huge nonfunctioning islet cell tumors, enhancement may be heterogeneous due to necrosis or cystic degeneration [8].

A similar CEUS pattern can also be observed in hypervascular metastases (from renal cell carcinoma and melanoma) [3].

c) Serous microcystic adenomas (SMAs) sometimes show at B-mode US a hypoechoic "solid-appearing" pattern, due to submacroscopic size of the cysts [10] (Fig. 4A); these tumors can be properly characterized by CEUS that demonstrates the typical honeycomb pattern (Fig. 4B), as well as by MR [11] (Figs. 4C and D).

\section{2) CYSTIC TUMORS AT B-MODE US}

Recently, a simple imaging-based classification of pancreatic cystic lesions into 4 types has been proposed: microcystic, unilocular, macrocystic, cystic with a solid component [12].

a) B-mode US can characterize a SMA when it demonstrates the typical honeycomb appearance $[10,12]$; CEUS, by depicting septa enhancement and the absence of papillary projections, allows a better diagnostic confidence (Fig. 5). In case of small nonsurgical lesions, CEUS doesn't need a further diagnostic step with CT and/or MR.

b) Unilocular single cyst (without internal septa, solid component, parietal or central calcifications) at B-mode US can be referred to pseudocyst, oligocystic serous cystadenoma, MCN, IPMT (branch-duct type) [12]; CEUS doesn't give any additional diagnostic information (Figs. 6A and B): in particular it is not able to find out communication with the pancreatic duct that can be demonstrated by MR cholangiopancreatography or CT, especially with curved reformatted images (Figs. 6C and D), in IPMTs [13].

Likewise, when two or more unilocular cysts are present, the differential diagnosis - restricted between pseudocysts and IPMTs [12] - cannot be made by CEUS and the resort to CT or MR is mandatory (Fig. 7).

c) Macrocystic lesions include multilocular cysts with fewer compartments than SMAs; besides, the compartments $(>2 \mathrm{~cm})$ are larger [12]; this category includes MCN and IPMT. A thick wall, thick septa and mural or septa calcifications are the most important findings associated with malignancy $[12,14]$. In these cases, although CEUS permits a better visualization of wall and septa [15], it does not give a significative diagnostic gain in comparison with B-mode US (Fig. 8).

d) Cysts with a solid component at B-mode US may be either unilocular or multilocular; true cystic tumors (MCN and IPMT) as well as solid tumors with a cystic component or cystic degeneration - primary or metastatic - are included in this category. All tumors of this group are either malignant or have a high malignant potential [12]; in these cases too CEUS does not offer a meaningful diagnostic contribution (Fig. 9).

\section{CONCLUSIONS}

As regards pancreatic hypoechoic lesions at B-mode US, CEUS - by detecting their vascularisation is a reliable imaging modality for their characterization. Sometimes, it may conclude the diagnostic work-up; however, in tumors judged resectable by CEUS, CT and/or MR are necessary for a more accu- 
rate evaluation of the local extension and metastatic spread, in order to confirm their resectability.

As regards cystic lesions at B-mode US, CEUS may give a higher diagnostic confidence, by depicting better septa and mural nodules, but in most cases it doesn't add significative information. Therefore, CT, MR or endoscopic US are almost always necessary for their characterization as well as for evaluation of their extension. Patient management depends on many factors (age, surgical risk, symptoms) as well as on imaging; in particular for small lesions (diameter $\leq 3$ $\mathrm{cm}$ ) without a solid component, which are very often benign, careful radiological follow-up is preferable to surgery [16].

\section{Conflict of Interest}

The authors have declared that no conflict of interest exists.

\section{References}

1. Lencioni R, Cioni D, Bartolozzi C. Tissue harmonic and contrast-specific imaging: back to gray scale in ultrasound. Eur Radiol 2002; 12:151-165.

2. Takeshima K, Kumada T, Toyoda H, et al. Comparison of iv contrast-enhanced sonography and histopathology of pancreatic cancer. AJR 2005; 185:1193-1200.

3. D'Onofrio M, Malagò R, Zamboni G, et al. Contrast-enhanced ultrasonography better identifies pancreatic tumor vascularization than helical CT. Pancreatology 2005; 5:398-402.

4. D'Onofrio M, Zamboni G, Tognolini A, et al. Mass-forming pancreatitis: value of contrast-enhanced ultrasonography. World J Gastroenterol 2006; 12:4181-4184.

5. Kim T, Muratami T, Takamura M, et al. Pancreatic mass due to chronic pancreatitis: correlation of $\mathrm{CT}$ and MR imaging features with pathologic findings. AJR 2001; 177:367-371.
6. Ozawa Y, Numata K, Tanaka K, et al. Contrast-enhanced sonography of small pancreatic mass lesions. J Ultrasound Med 2002; 21:983-991.

7. Rickes S, Unkrodt K, Neye K, Wermke O, Wermke W. Differentiation of pancreatic tumors by conventional ultrasound, unenhanced and echo-enhanced power Doppler sonography. Scand J Gastroenterol 2002; 37:1313-1320.

8. D'Onofrio M, Mansueto G, Falconi M, Procacci C. Neuroendocrine pancreatic tumor: value of contrast enhanced ultrasonography. Abdom Imaging 2004; 29:246-258.

9. Sheth S, Hruban RK, Fishman E. Helical CT of islet cell tumors of the pancreas: typical and atypical manifestations. AJR 2002; 179:725-730.

10. Fugazzola C, Procacci C, Bergamo Andreis IA, et al. Cystic tumors of the pancreas: evaluation by ultrasonography and computed tomography. Gastrointest Radiol 1991; 16:53-61.

11. Procacci C, Carbognin G, Biasutti C, et al. Serous cystadenoma of the pancreas: imaging findings. Radiol Med 2001; 102:23-31.

12. Sahani DV, Kadavigere R, Saokar A, Fernandez-del Castillo C, Brugge W, Hahn P. Cystic pancreatic lesions: a simple imaging-based classification system for guiding management. Radiographics 2005; 25:1471-1484.

13. Procacci C, Carbognin G, Biasiutti C, Guarise A, Ghirardi C, Schenal G. Intraductal papillary mucinous tumors of the pancreas: spectrum of CT and MR findings with pathologic correlation. Eur Radiol 2001; 11:1939-51.

14. Procacci C, Carbognin G, Accordini S, et al. CT features of malignant mucinous cystic tumors of the pancreas. Eur Radiol 2001; 11:1626-1630.

15. Itoh $\mathrm{T}$, Hirooka $\mathrm{Y}$, Itoh $\mathrm{A}$, et al. Usefulness of contrast-enhanced transabdominal ultrasonography in the diagnosis of intraductal papillary tumors of the pancreas. Am J Gastroenterol 2005; 100:144-152.

16. Sahani DV, Saokar A, Hahn PF, Brugge WR, Fernandez-del Castillo C. Pancreatic cysts $3 \mathrm{~cm}$ or smaller: how aggressive should treatment be? Radiology 2006; 238:912-919.

\section{Figures}

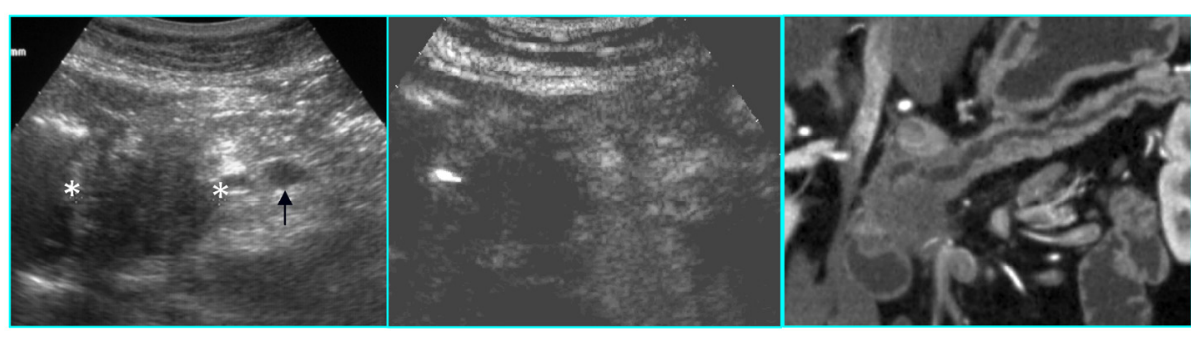

A

B

C

Fig. 1.- Adenocarcinoma in a 85-year-old woman who presented with diabetes at sudden onset. A. B-mode US (transverse scan) shows a hypoechoic mass, located in the head of the pancreas (asterisks), $3.5 \mathrm{~cm}$ in diameter. Splenic vein (arrow) is visible. B. On CEUS the mass is hypoechoic (asterisks) compared to the surrounding parenchyma in the arterial phase. C. MDCT curved reformatted image depicts pancreatic duct dilatation and hypodense lesion in the head of the pancreas. 


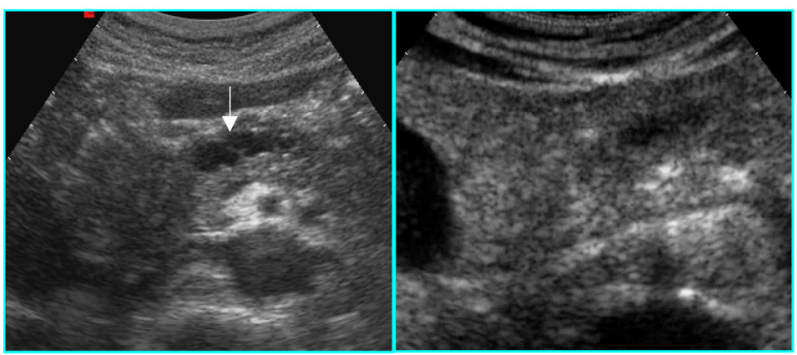

A

Fig 2.- Adenocarcinoma in a 45-year-old woman who presented with jaundice. A. B-mode US (trasverse scan) shows a hypoechoic mass in the head of the pancreas, $4 \mathrm{~cm}$ in diameter. Main pancreatic duct dilatation (arrow) is visible. B. On CEUS the mass is isoechoic in the arterial phase.

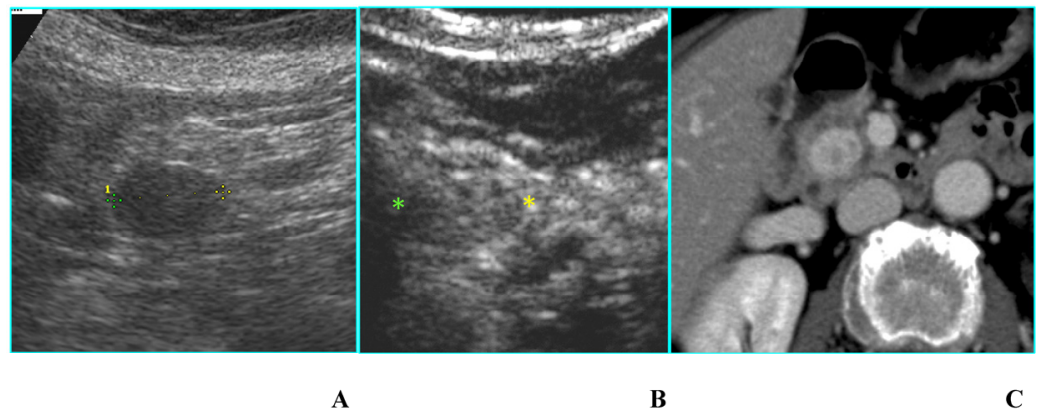

Fig 3- Nonfunctioning islet cell tumor in an asymptomatic 80-year-old woman. A. B-mode US shows a 2-cm hypoechoic nodule (calipers) located in the uncinate process of the pancreas. B. On CEUS the nodule is hyperechoic (asterisks) in the portal phase. C. Axial CT image depicts the same nodule as hyperdense in the venous phase (arrow).

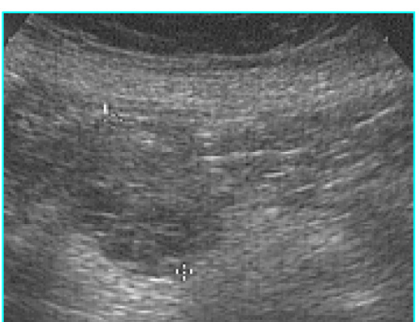

A

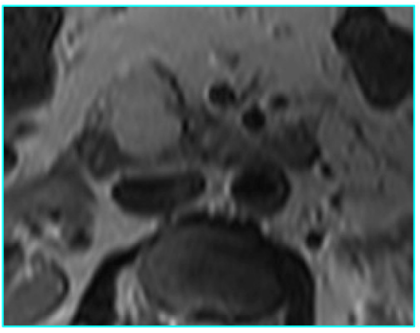

C

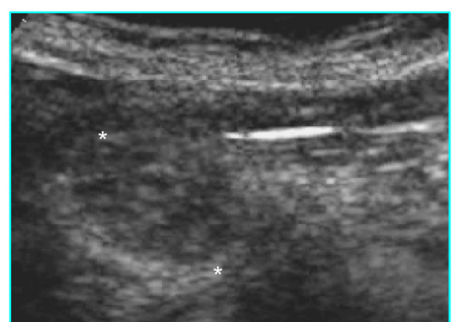

B

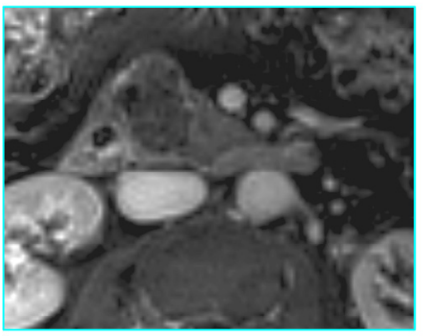

D

Fig 4- Serous microcystic adenoma in an asymptomatic 66-year-old woman. A. B-mode US shows a hypoechoic mass (calipers) located in the head of the pancreas. B. On CEUS the lesion has a honeycomb appearance (asterisks), typical of serous microcystic adenoma. C. Axial T2-weighted MR image shows a hyperintese lesion made up of cluster of microcysts. D. Axial gadolinium-enhanced fat-suppressed T1-weighted MR image depicts the honeycomb appearance with enhancement of thin septa. 

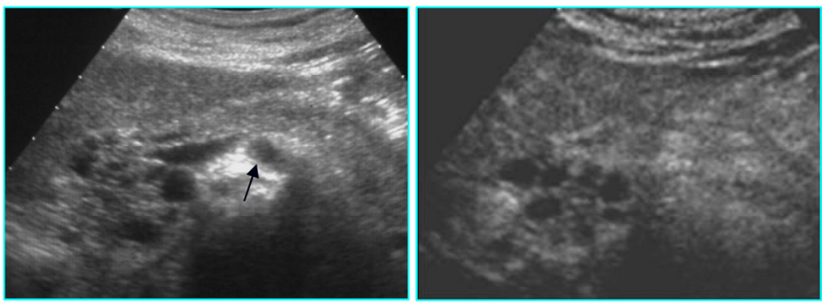

A

Fig 5- Serous microcystic adenoma in an asymptomatic 82-year-old woman. A. B-mode US shows a focal lesion located in the head of the pancreas with a honeycomb appearance. Superior mesenteric artery (arrow) is visible. B. CEUS confirms the findings of B-mode US, allowing a better diagnostic confidence.

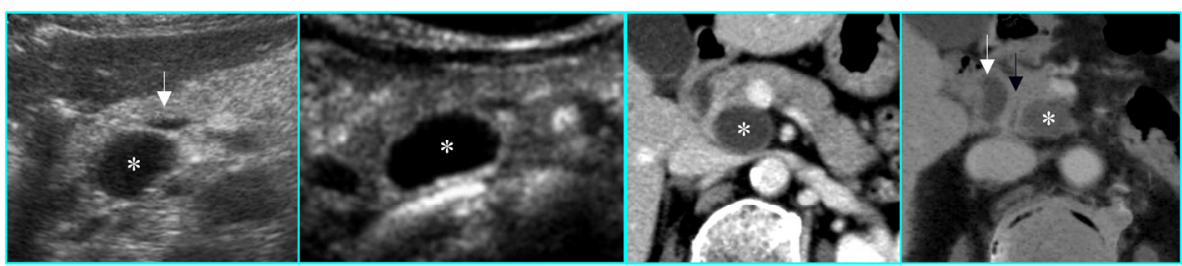

A

B

C

D

Fig 6- Benign mucinous cystic neoplasm in a 79 old-woman with history of abdominal pain. A. B-mode US shows a unilocular cyst $3,5 \mathrm{~cm}$ in diameter (asterisk), located in the uncinate process of the pancreas; main pancreatic duct, not dilated (white arrow), is visible. B. CEUS image confirms the unilocular aspect of the cyst (asterisk), without septa or mural nodules. C and D. MDCT confirms on axial image (C) the unilocular aspect of the cyst (asterisk) and on MinIP image (D) demonstrates the absence of communication with the pancreatic duct (black arrow); dilated bile duct (white arrow) is visible.

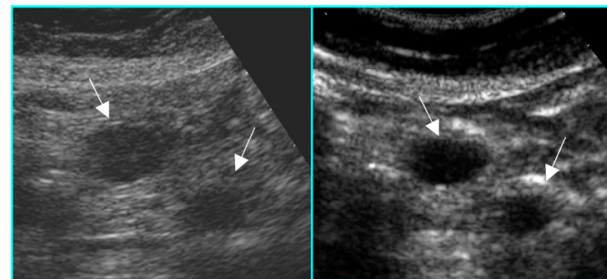

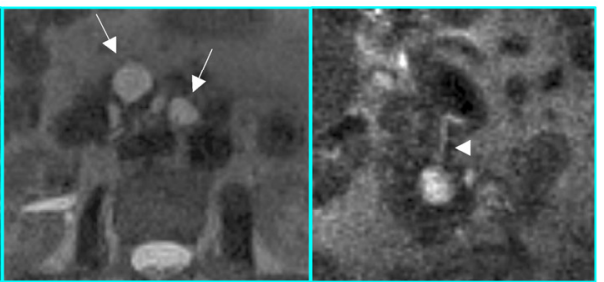

C

Fig 7- Double benign intraductal papillary mucinous tumor in an asymptomatic 72-year-old woman. A. B-mode US shows two simple cysts (arrows) located in the head of the pancreas. B. CEUS confirms the presence of two simple cysts without septa or mural nodules (arrows). C and D. MR on axial T2-weighted image (C) confirms the unilocular aspect of the cysts (arrows) and on coronal T2-weighted image (D) well demonstrates the communication of the greater cyst with the pancreatic duct (arrowhead).

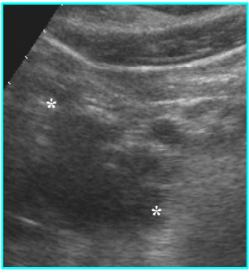

$\mathbf{A}$

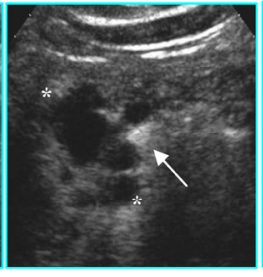

$\mathbf{B}$

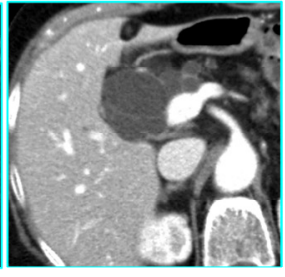

C

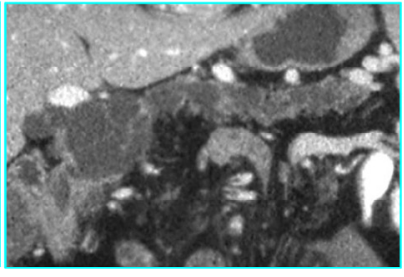

D

Fig. 8 - Benign intraductal papillary mucinous tumor in an asymptomatic 81-year-old woman. A. B-mode US shows a huge multilocular cyst (calipers) with compartments $>2 \mathrm{~cm}$ in diameter located in the head of the pancreas. B. CEUS better shows thin septa and absence of papillary projections in the lesion (calipers). Portal vein (arrow) is filled with contrast agent. C and D. MDCT on axial image (C) confirms a macrocystic lesion with thin septa; on curved reformatted image (D) the communication with the pancreatic duct is demonstrated. 


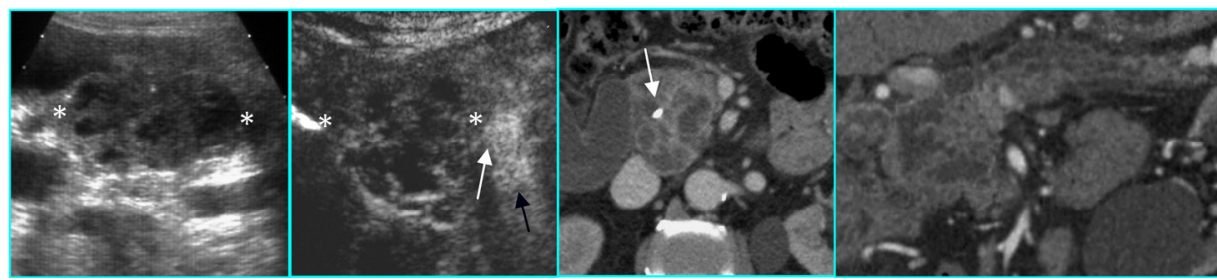

A

C

D

Fig 9- Malignant intraductal papillary mucinous tumor in a 80-year-old man who presented with obstructive jaundice. A. B-mode US shows a multilocular cystic lesion with mural nodules (calipers) located in the head of the pancreas. B. CEUS well depicts the vascularized septa and nodules in the lesion (calipers). Superior mesenteric artery (black arrow) and superior mesenteric vein (white arrow) are filled with contrast agent. C and D. MDCT on axial image (C) shows a multilocular lesion with a solid component; a stent is inserted in the common bile duct (arrow); on the curved reformatted image (D) the communication of the tumor with the pancreatic duct is demonstrated. 